\title{
A Fast Fourier Transform Technique for Pricing European Options with Stochastic Volatility and Jump Risk
}

\author{
Su-mei Zhang ${ }^{1,2}$ and Li-he Wang ${ }^{3}$ \\ ${ }^{1}$ School of Science, Xi'an Jiaotong University, Xi'an 710049, China \\ ${ }^{2}$ School of Science, Xi'an University of Posts and Telecommunications, Xi'an 710121, China \\ ${ }^{3}$ Department of Mathematics, The University of Iowa, Iowa City, IA 52242, USA
}

Correspondence should be addressed to Su-mei Zhang, zhanggsumei@sina.com

Received 7 April 2011; Revised 15 October 2011; Accepted 31 October 2011

Academic Editor: M. D. S. Aliyu

Copyright (c) 2012 S.-m. Zhang and L.-h. Wang. This is an open access article distributed under the Creative Commons Attribution License, which permits unrestricted use, distribution, and reproduction in any medium, provided the original work is properly cited.

We consider European options pricing with double jumps and stochastic volatility. We derived closed-form solutions for European call options in a double exponential jump-diffusion model with stochastic volatility(SVDEJD). We developed fast and accurate numerical solutions by using fast Fourier transform (FFT) technique. We compared the density of our model with those of other models, including the Black-Scholes model and the double exponential jump-diffusion model. At last, we analyzed several effects on option prices under the proposed model. Simulations show that the SVDEJD model is suitable for modelling the long-time real-market changes and stock returns are negatively correlated with volatility. The model and the proposed option pricing method are useful for empirical analysis of asset returns and managing the corporate credit risks.

\section{Introduction}

The classical Black-Scholes (BS) model [1] has long been known to result in systematically biased option valuation. By adding jumps to the archetypal price process with Gaussian innovations Merton [2] is able to partly explain the observed deviations from the benchmark model which are characterized by fat tail and excess kurtosis in the returns distribution. For an overview of "stylized facts" on asset returns see Cont [3]. Statistical properties of implied volatilities are summarized in Cont et al. [4]. In the sequel also other authors develop more realistic models, for example, the pure jump models of Eberlein and Keller [5], Madan et al. [6], and Duffie et al. [7], stochastic volatility models of Steven [8], and stochastic volatility model with normal jumps of Bates [9] and Keppo et al. [10]. The double exponential jump-diffusion (DEJD) model, recently proposed by Kou [11], generates a highly skewed and leptokurtic distribution and is capable of matching key features of stock and index 
returns. Moreover, the DEJD model leads to tractable pricing formulas for exotic and path dependent options [12]. Accordingly, the DEJD model has gained wide acceptance. However, the DEJD model cannot capture the volatility clustering effects, which can be captured by stochastic volatility models [13]. Jump-diffusion models and the stochastic volatility model complement each other: the stochastic volatility model can incorporate dependent structures better, while the DEJD model has better analytical tractability, especially for path-dependent options. Since allowing interest rates to be stochastic does not improve pricing performance any further [14], the model that combines stochastic volatility and double exponential jumpdiffusion (SVDEJD) may be more reasonable.

In the BS setting, the probability measure has a well-known analytic form [15], but, under stochastic volatility, it can only be obtained numerically. Monte Carlo simulation and the finite difference method are usually used to value the options. But, the two techniques require substantially more computing time and thus are difficult to be applied in real option pricing. Recently, being fast, accurate, and easy to implement, Fourier transforms have been widely used in valuing financial derivatives, for example, Carr and Madan [16] propose Fourier transforms with respect to log-strike price; Duffie et al. [7] offer a comprehensive survey that the Fourier methods are applicable to a wide range of stochastic processes; Carr and $\mathrm{Wu}$ [17] apply the transforms to time-changed Lévy processes and the class of generalized affine models. Hurd and Zhou [18] express the spread option payoff in terms of the gamma function and FFT technique. For an overview of option pricing using Fourier transforms, see Schmelzle [19].

The current paper extends the study of option pricing under the DEJD model in three ways. First, we propose a model which combines the double jumps and stochastic volatility. Second, using the martingale method, Fourier transform formula, and FeynmanKac theorem, we obtain a closed-form solution for European call options pricing under the proposed model. Third, we obtain fast and accurate numerical solutions for European call options pricing by FFT technique.

The rest of the paper is organized as follows. Section 2 develops the underlying pricing model. Section 3 derives a closed-form solution for European call options pricing under the proposed model. Section 4 provides approximation solutions for European call options pricing by FFT technique. Section 5 numerically compares the density of the solutions to the alternative models and analyzes several effects on potion prices. Section 6 concludes. Applied program codes in Matlab package are presented in the appendix.

\section{The Model}

We consider an arbitrage-free, frictionless financial market where only riskless asset $B$ and risky asset $S$ are traded continuously up to a fixed horizon date $T$. Let $\left\{\Omega, \mathcal{F},\left\{\mathcal{F}_{t}\right\}_{0 \leq t \leq T}, P\right\}$ be a complete probability space with a filtration satisfying the usual conditions, that is, the filtration is continuous on the right and $\mathcal{F}_{0}$ contains all $P$-null sets. Suppose $W(t), W_{v}(t)$ are both standard Brownian motion, which is $\mathcal{F}_{t}$ adapted, and $W(t)$ has correlation $\rho$ with $W_{v}(t)$.

Let $S(t)$ represent the price for a stock or a stock portfolio. Generally, instantaneous variance of asset returns in financial markets shows randomness; thus, the continuous part of the price process, defined as $S^{c}(t)$, is

$$
d S^{c}(t)=r S^{c}(t) d t+\sigma \sqrt{V(t)} S^{c}(t) d W(t)
$$


where $r$ is risk-free rate and $\sigma$ is nonnegative constant, and suppose $S^{c}(0)=s$, which can be set equal to 1 without any loss of generality. The size of the diffusion component is determined by $V(t)$, which represents, absent of any jump occurring, the level of (stochastic) return variance attributable to diffusion variations. For tractability, let $V(t)$ follow a squareroot process:

$$
d V(t)=\left(\theta_{v}-\alpha_{v} V(t)\right) d t+\sigma_{v} \sqrt{V(t)} d W_{v}(t)
$$

where nonnegative constants $\theta_{v}, \theta_{v} / \alpha_{v}$, and $\sigma_{v}$, respectively, reflect the speed of adjustment, the long-run mean, and the variation coefficient of $V(t)$, and suppose $V(0)=V_{0}$.

It has been suggested from extensive empirical studies that markets tend to have both overreaction and underreaction to various good or bad news. One may interpret the jump part of the model as the market response to outside news. Good or bad news arrives according to a Poisson process, and the asset price changes in response according to the jump size distribution. According to Kou [11], the jumps in the log-price are modeled as a sequence of i.i.d. nonnegative random variables that occur at times determined by an independent Poisson process $N(t)$ with constant intensity $\lambda>0$ such that $Y=\ln U$ has an asymmetric double exponential distribution with the density

$$
f_{Y}(y)=p \eta_{1} e^{-\eta_{1} y} \mathbf{1}_{y \geq 0}+q \eta_{2} e^{\eta_{2} y} \mathbf{1}_{\mathrm{y}<0,}, \quad \eta_{1}>1, \eta_{2}>0,
$$

where 1 denotes the indicator function, so $1_{y} \geq 0$ equals 1 if $y \geq 0$, but 0 otherwise. $p, q \geq$ $0, p+q=1$ are up-move jump and down-move jump, respectively. Except for $W(t)$ which has correlation with $W_{v}(t)$, all sources of randomness, $W(t), W_{v}(t), N(t), Y_{j}$, and $N(t)$, are assumed to be independent.

Because of jumps and stochastic volatility, the risk-neutral probability measure is not unique. Following Naik and Lee [20] and Kou [11], by using the rational expectations argument with a HARA-type utility function for the representative agent, one can choose a particular risk-neutral measure $P^{*}$ so that the equilibrium price of an option is given by the expectation under this risk-neutral measure of the discounted option payoff. Throughout this paper, we assume that there exists a martingale probability measure $P^{*}$ being equivalent to $P$. Let $X(t)$ be the sum of all the jumps which occur up to and including time $t, J(t)=$ $\exp [X(t)]-E[\exp (X(t))]$, we have

$$
J(t)=\exp \left[X(t)-\lambda t\left(\frac{p \eta_{1}}{\eta_{1}-1}+\frac{q \eta_{2}}{\eta_{2}+1}-1\right)\right] .
$$

Obviously, $J(t)$ is a $P$-martingale. Finally, the price process $S(t)$ is defined as

$$
S(t)=S^{c}(t) J(t)
$$

Remark 2.1. The model contains most existing models as special cases. For example, we obtain (1) the BS model by setting $\lambda=0$ and $\theta_{v}=\alpha_{v}=\sigma_{v}=0$; (2) the SV model by setting $\lambda=0$;

(3) the DEJD model by setting $\theta_{v}=\alpha_{v}=\sigma_{v}=0$. 
Let $d W(t)=\rho d W_{v}(t)+\sqrt{1-\rho^{2}} d Z(t)$, where $Z(t)$ is standard Brownian motion that is $F_{t}$ adapted, and independent of $W_{v}(t), N(t)$, and random variables $U_{j}$. From Itŏ's formula, we have

$$
\begin{aligned}
\ln S(t)= & \ln J(t)+\ln S^{c}(t) \\
= & X(t)-\lambda t\left(\frac{p \eta_{1}}{\eta_{1}-1}+\frac{q \eta_{2}}{\eta_{2}+1}-1\right)+r t \\
& +\left[\rho \sigma \int_{0}^{t} \sqrt{V(t)} d W_{v}(t)-\frac{1}{2} \sigma^{2} \rho^{2} \int_{0}^{t} V(t) d t\right] \\
& +\left[\sqrt{1-\rho^{2}} \sigma \int_{0}^{t} \sqrt{V(t)} d Z(t)-\frac{1}{2} \sigma^{2}\left(1-\rho^{2}\right) \int_{0}^{t} V(t) d t\right] \\
= & X(t)-\lambda t\left(\frac{p \eta_{1}}{\eta_{1}-1}+\frac{q \eta_{2}}{\eta_{2}+1}-1\right)+r t+\xi_{t}+\varsigma_{t} .
\end{aligned}
$$

\section{A Closed-Form Solution of European Option Pricing}

In this section, we derive closed-form solution of a European call option pricing under the SVDEJD model. For a European put option, we can obtain easily corresponding result by the put-call parity [1]. For this purpose, we need the following results.

Lemma 3.1. Supposing the variance process $V(t)$ follows (2.2) and $s_{1}, s_{2}$ are any complex, one has

$$
E\left\{\exp \left[-s_{1} \int_{0}^{T} V(t) d t-s_{2} V(t)\right]\right\}=\exp \left[A(T)-B(T) V_{0}\right]
$$

where

$$
\begin{gathered}
A(T)=\frac{2 \theta_{v}}{\sigma_{v}^{2}} \ln \left[\frac{2 \gamma e^{(1 / 2)\left(\alpha_{v}-\gamma\right) T}}{2 \gamma e^{-\gamma T}+\left(\alpha_{v}+\gamma+\sigma_{v}^{2} s_{2}\right)\left(1-e^{-\gamma T}\right)}\right], \\
B(T)=\frac{\left(1-e^{-\gamma T}\right)\left(2 s_{1}-\alpha_{v} s_{2}\right)+\gamma s_{2}\left(1+e^{-\gamma T}\right)}{2 \gamma e^{-\gamma T}+\left(\alpha_{v}+\gamma+\sigma_{v}^{2} s_{2}\right)\left(1-e^{-\gamma T}\right)}, \\
\gamma=\sqrt{\alpha_{v}^{2}+2 \sigma_{v}^{2} s_{1}} .
\end{gathered}
$$

Proof. Let $F(V, 0, T)=E\left\{\exp \left[-s_{1} \int_{0}^{T} V(t) d t-s_{2} V(t)\right]\right\}$. Because of the affine structure of the variance process (2.2), we obtain that $F(V, 0, T)$ has a solution of the following form:

$$
F(V, 0, T)=\exp \left[A(T)-B(T) V_{0}\right]
$$


From the Feynman-Kac formula, $F(V, 0, T)$ is the solution of the following backward Parabolic partial differential equation with the Cauchy problem:

$$
\begin{gathered}
\frac{\partial F}{\partial t}+\left(\theta_{v}-\alpha_{v} V\right) \frac{\partial F}{\partial V}+\frac{1}{2} \sigma_{v}^{2} V \frac{\partial^{2} F}{\partial V^{2}}-s_{1} V F=0 \\
F(V, 0,0)=\exp \left(-s_{2} V_{0}\right)
\end{gathered}
$$

Putting (3.3) in (3.4), we have

$$
\begin{gathered}
A_{t}(T)-\theta_{v} B(T)=0, \quad A(0)=0, \\
-B_{t}(T)+\frac{1}{2} \sigma_{v}^{2} B^{2}(T)+\alpha_{v} B(T)-s_{1}=0, \quad B(0)=s_{2} .
\end{gathered}
$$

Solving (3.5), we can obtain the result of Lemma 3.1.

Lemma 3.2. Supposing the asset price $S(T)$ follows (2.6) and $z$ is any complex, one has

$$
\begin{aligned}
E\{\exp [-r T+z \ln S(T)]\}=\exp \{( & z-1) r T+\lambda T\left(\frac{p \eta_{1}}{\eta_{1}-z}+\frac{q \eta_{2}}{\eta_{2}+z}-1\right) \\
& -z \lambda T\left(\frac{p \eta_{1}}{\eta_{1}-1}+\frac{q \eta_{2}}{\eta_{2}+1}-1\right)-z \frac{\rho \sigma}{\sigma_{v}}\left(V(T)+\theta_{v} T\right) \\
& +\frac{2 \theta_{v}}{\sigma_{v}^{2}} \ln \left[\frac{2 \gamma e^{(1 / 2)\left(\alpha_{v}-\gamma\right) T}}{2 \gamma e^{-\gamma T}+\left(\alpha_{v}+\gamma+\sigma_{v}^{2} s_{2}\right)\left(1-e^{-\gamma T}\right)}\right] \\
& \left.-\frac{\left(1-e^{-\gamma T}\right)\left(2 s_{1}-\alpha_{v} s_{2}\right)+\gamma s_{2}\left(1+e^{-\gamma T}\right)}{2 \gamma e^{-\gamma T}+\left(\alpha_{v}+\gamma+\sigma_{v}^{2} s_{2}\right)\left(1-e^{-\gamma T}\right)} V_{0}\right\},
\end{aligned}
$$

where

$$
s_{1}=-(z-1) z \frac{1}{2} \sigma^{2}\left(1-\rho^{2}\right)-z\left(\frac{\rho \sigma}{\sigma_{v}} \alpha_{v}-\frac{1}{2} \sigma^{2} \rho^{2}\right), \quad s_{2}=-z \frac{\rho \sigma}{\sigma_{v}} .
$$

Proof. Let $\phi(z)=E\{\exp [-r T+z \ln S(T)]\}$. Because $N(t)$ is independent of $W(t), W_{v}(t)$, and $Z(t)$, we have

$$
\phi(T)=e^{(z-1) r T} E\left[e^{z \ln J(T)}\right] E\left[e^{z\left(\xi_{T}+\zeta_{T}\right)}\right]=e^{(z-1) r T} C(T) D(T)
$$

From (2.2) and (2.3), we have

$$
C(T)=\exp \left[\lambda T\left(\frac{p \eta_{1}}{\eta_{1}-z}+\frac{q \eta_{2}}{\eta_{2}+z}-1\right)-z \lambda T\left(\frac{p \eta_{1}}{\eta_{1}-1}+\frac{\mathrm{q} \eta_{2}}{\eta_{2}+1}-1\right)\right] .
$$


Because $W_{v}(t)$ is a standard Brownian motion, we have

$$
E\left(\varsigma_{T}\right)=-\frac{1}{2} \sigma^{2}\left(1-\rho^{2}\right) \int_{0}^{T} V(T) d t, \operatorname{Var}\left(\varsigma_{T}\right)=\sigma^{2}\left(1-\rho^{2}\right) \int_{0}^{T} V(T) d t .
$$

Then,

$$
\begin{aligned}
D(T)= & E\left\{\exp \left[(z-1) z \frac{1}{2} \sigma^{2}\left(1-\rho^{2}\right) \int_{0}^{T} V(t) d t+z \xi_{T}\right]\right\} \\
= & \exp \left\{-z \frac{\rho \sigma}{\sigma_{v}}\left[V(T)+\theta_{v} T\right]\right\} \\
& \times E\left\{\exp \left[(z-1) z \frac{1}{2} \sigma^{2}\left(1-\rho^{2}\right)+z\left(\frac{\rho \sigma}{\sigma_{v}} \alpha_{v}-\frac{1}{2} \sigma^{2} \rho^{2}\right)\right] \int_{0}^{T} V(t) d t-z \frac{\rho \sigma}{\sigma_{v}} V_{0}\right\} .
\end{aligned}
$$

Let $s_{1}=-(z-1) z(1 / 2) \sigma^{2}\left(1-\rho^{2}\right)-z\left(\left(\rho \sigma / \sigma_{v}\right) \alpha_{v}-(1 / 2) \sigma^{2} \rho^{2}\right)$, and $s_{2}=-z\left(\rho \sigma / \sigma_{v}\right)$. From Lemma 3.1, we have

$$
\begin{aligned}
D(T)=\exp \{- & -z \frac{\rho \sigma}{\sigma_{v}}\left(\mathrm{~V}(T)+\theta_{v} T\right)+\frac{2 \theta_{v}}{\sigma_{v}^{2}} \ln \left[\frac{2 \gamma e^{(1 / 2)\left(\alpha_{v}-\gamma\right) T}}{2 \gamma e^{-\gamma T}+\left(\alpha_{v}+\gamma+\sigma_{v}^{2} s_{2}\right)\left(1-e^{-\gamma T}\right)}\right] \\
& \left.-\frac{\left(1-e^{-\gamma T}\right)\left(2 s_{1}-\alpha_{v} s_{2}\right)+\gamma s_{2}\left(1+e^{-\gamma T}\right)}{2 \gamma e^{-\gamma T}+\left(\alpha_{v}+\gamma+\sigma_{v}^{2} s_{2}\right)\left(1-e^{-\gamma T}\right)} V_{0}\right\} .
\end{aligned}
$$

From (3.8), (3.9), (3.10) and (3.12), we can obtain the required Lemma 3.2.

Lemma 3.3. Suppose $\varphi(u)=E[\exp (i u \ln S(T)]$ is the characteristic function of $\ln S(T)$; then

$$
\begin{aligned}
\varphi(u)= & {\left[\frac{2 \delta}{2 \delta+\left(\alpha_{v}-\delta-i u \rho \sigma \sigma_{v}\right)\left(1-e^{-\delta T}\right)}\right]^{2 \theta_{v} / \sigma_{v}^{2}} } \\
& \times \exp \left\{i u \ln S(t)+\frac{\theta_{v}\left(\alpha_{v}-\delta\right) T}{\sigma_{v}^{2}}-\frac{i u \theta_{v} \sigma \rho T}{\sigma_{v}}\right. \\
& +\lambda T\left[\frac{p \eta_{1}}{\eta_{1}-i u}+\frac{q \eta_{2}}{\eta_{2}+i u}-1-i u\left(\frac{p \eta_{1}}{\eta_{1}-1}+\frac{q \eta_{2}}{\eta_{2}+1}-1\right)\right] \\
& \left.+i u r T+\epsilon V_{0}\right\},
\end{aligned}
$$

where

$$
\begin{aligned}
& \delta=\sqrt{\left(\alpha_{v}-i u \rho \sigma \sigma_{v}\right)^{2}+i u(1-i u) \sigma^{2} \sigma_{v}^{2}} \\
& \epsilon=\frac{i u(i u-1) \sigma^{2}\left(1-e^{-\delta T}\right)}{2 \delta+\left(\alpha_{v}-\delta-i u \rho \sigma \sigma_{v}\right)\left(1-e^{-\delta T}\right)} .
\end{aligned}
$$


Proof. Let $\phi(z)=E\{\exp [-r T+z \ln S(T)]\}$. Because

$$
\varphi(u)=E[\exp (i u \ln S(T))]=\frac{E\{\exp [-r T+i u \ln S(T)]\}}{E[\exp (-r T)]}=\frac{\phi(i u)}{\phi(0)},
$$

from Lemma 3.2, we can obtain the required Lemma 3.3.

Theorem 3.4. Let $k$ denote the $\log$ of the strike price $K, x_{T}=\ln (S(T))$, and $C_{T}(k)$ the desired value of a T-maturity call option with strike $\exp (k)$. Assume that, under $P^{*}$, the underlying nondividendpaying stock price $S(t)$ and its components are given by $(2.1)-(2.5), \varphi(u)$ is the characteristic function of $x_{T}, q(x)$ is the density of $x_{T}$; then the initial call value $C_{T}(k)$ is written as

$$
\begin{aligned}
C_{T}(k)= & \frac{1}{2}\left(S(t)-e^{-r T} K\right) \\
& +\frac{1}{\pi} \int_{0}^{\infty} S(t) \Re\left[\frac{e^{i u k} \varphi_{T}(u-i)}{i u}\right]-e^{-r T} K \Re\left[\frac{e^{i u k} \varphi_{T}(u)}{i u}\right] d u,
\end{aligned}
$$

where $\mathfrak{R}[\cdot]$ represents real part.

Proof. From the risk-neutral theory, we have

$$
\begin{aligned}
C_{T}(k) & =E\left[e^{-r T}(S(T)-K)^{+}\right] \\
& =e^{-r T} \int_{0}^{+\infty}(S(T)-K)^{+} q(S(T)) d S(T) \\
& =e^{-r T} \int_{k}^{+\infty} e^{x_{T}} q(x) d x-e^{-r T} K \int_{k}^{+\infty} q(x) d x \\
& =S \Pi_{1}-e^{-r T} \Pi_{2} .
\end{aligned}
$$

Introducing a change of measure from $P^{*}$ to $Q^{*}$ by a Radon-Nikodym derivative, we get

$$
\frac{d Q^{*}}{d P^{*}}=\frac{e^{x_{T}}}{E\left[e^{x_{T}}\right]}
$$

With this new measure $Q^{*}$, the Fourier transform of $\Pi_{1}$ is defined as

$$
E^{Q^{*}}\left[e^{i u x_{T}}\right]=\frac{\varphi(u-i)}{\varphi(-i)} .
$$

Because of the no-arbitrage condition, we can obtain

$$
\Pi_{1}=\frac{1}{2}+\frac{1}{\pi} \int_{0}^{\infty} \mathfrak{R}\left[\frac{e^{-i u k} \varphi_{T}(u-i)}{i u \varphi_{T}(-i)}\right] d u .
$$


From the Fourier transform formula, the probability density for our model is given by

$$
q(x)=\frac{1}{\pi} \int_{0}^{+\infty} e^{-i u k} \varphi(u) d u .
$$

Hence,

$$
\Pi_{2}=\int_{k}^{+\infty}\left(\frac{1}{\pi} \int_{0}^{+\infty} e^{-i u k} \varphi(u) d u\right) d x
$$

Changing the order of integration, we have

$$
\Pi_{2}=\frac{1}{2}+\frac{1}{\pi} \int_{0}^{\infty} \mathfrak{R}\left[\frac{e^{-i u k} \varphi_{\mathrm{T}}(u)}{i u}\right] d u
$$

From (3.17), (3.20), and (3.23), we can obtain the required Theorem 3.4.

Remark 3.5. In (3.16), $C_{T}(k)$ tends to $S_{0}$ not zero as $k$ goes to $-\infty$. Hence, $C_{T}(k)$ is not $L^{1}$ (absolutely integrable) and a Fourier transform does not exist.

\section{Fast Fourier Transform for European Option Pricing}

Since the integrand in (3.16) is singular at the required evaluation point $u=0$, the FFT cannot be applied directly to evaluate the integrals we mentioned above. Therefore, instead of solving for the risk-neutral exercise probabilities of finishing in-the-Money (ITM), Carr and Madan [16] introduce a new technique with the key idea to calculate the Fourier transform of a modified call option price with respect to the logarithmic strike price. With this specification and a FFT routine, a whole range of option prices can be obtained within a single Fourier inversion. In this section, we develop the numerical solutions of the prices by using the idea of Carr and Madan [16].

\subsection{Fourier Transform of ITM and at-the-Money (ATM) Option Prices}

By introducing an exponential damping factor $e^{\alpha k}$ with $\alpha>0$, it is possible to make the integrand in (3.16) be square integrable. We modified the pricing function (3.16) by

$$
C_{T}(k)=\frac{\exp (-\alpha k)}{\pi} \int_{0}^{\infty} e^{-i v k} \psi_{T}(v) d v
$$

where $\psi_{T}(v)=e^{-r T} \varphi_{T}(v-(\alpha+1) i) /\left(\alpha^{2}+\alpha-v^{2}+i(2 \alpha+1) v\right)$.

This method is viable when $\alpha$ is chosen in a way that the damped option price is well behaved. Damping the option price with $e^{\alpha k}$ makes it integrable for the negative axis $k<0$. On the other hand, for $k>0$ the option prices increase by the exponential $e^{\alpha k}$, which 
influences the integrability for the positive axis. A sufficient condition of $c_{T}(k)$ to be integrable for both sides (square integrability) is given by $\psi(0)$ being finite, that is,

$$
\psi(0)=\frac{e^{-r T} \varphi_{T}(-(\alpha+1) i)}{\alpha^{2}+\alpha}<\infty
$$

Thus we need $\varphi_{T}(-(\alpha+1) i)<\infty$, which is equivalent to

$$
E^{Q}\left[S(T)^{1+\alpha}<\infty\right]
$$

Therefore, $c_{T}(k)$ is well behaved when the moments of order $1+\alpha$ of the underlying asset exist and are finite. If not all moments of $S(T)$ exist, this will impose an upper bound on $\alpha$. We find that one quarter of this upper bound serves as a good choice for $\alpha$.

Using the trapezoid rule for the integral on the right-hand side of (4.1) and setting $v_{j}=\eta(j-1)$, an approximation for $C_{T}(k)$ is

$$
C_{T}(k) \approx \frac{\exp (-\alpha k)}{\pi} \sum_{j=1}^{N} e^{-i v_{j} k} \psi_{T}\left(v_{j}\right) \eta
$$

The FFT returns $N$ values of $k$, and we employ a regular spacing of size $h$ so that our values for $k$ are

$$
k_{u}=-b+h(u-1) \text { for } u=1, \ldots, N \text {. }
$$

This gives us log-strike levels ranging from $-b$ to $b$, where

$$
b=\frac{1}{2} N h .
$$

In order to apply FFT we define

$$
\eta h=\frac{2 \pi}{N}
$$

To obtain an accurate integration with larger values of $\eta$, we incorporate Simpson's rule weightings into our summation. From (4.1)-(4.7)and Simpson's rule weightings, we obtain ATM and ITM call value as

$$
C\left(k_{u}\right)=\frac{\exp \left(-\alpha k_{u}\right)}{\pi} \sum_{j=1}^{N} e^{-i(2 \pi / N)(j-1)(i-1)} e^{i b v_{j}} \psi\left(v_{j}\right) \frac{\eta}{3}\left[3+(-1)^{j}-\omega_{j-1}\right],
$$

where $\omega_{n}$ is the Kronecker delta function that is unity for $n=0$ and zero otherwise. The summation in (4.8) is an exact application of the FFT. 


\subsection{Fourier Transform of out-of-the-Money (OTM) Option Prices}

In the previous section call values are calculated by an exponential function to obtain square integrable function whose Fourier transform is an analytic function of the characteristic function of the log-price. But, for very short maturities, the call value approaches its non analytic intrinsic value causing the integrand in the Fourier inversion to be high oscillate, and therefore difficult to integrate numerically. We introduce an alternative approach that works with time values only, which is quite similar to the previous approach. But in this case the call price is obtained via the Fourier transform of a modified time value, where the modification involves a hyperbolic sine function instead of an exponential function.

Let $z_{T}(k)$ denote the time value of an OTM option, that is, for $k<x_{T}$ we have the put price for $z_{T}(k)$ and for $k<x_{T}$ we have the call price. Scaling $S_{0}=1$ for simplicity, $z_{T}(k)$ is defined by

$$
z_{T}(k)=e^{-r T} \int_{-\infty}^{\infty}\left[\left(e^{k}-e^{x_{T}}\right) \mathbf{1}_{x_{T}<k, k<0}+\left(e^{x_{T}}-e^{k}\right) \mathbf{1}_{x_{T}>k, k>0}\right] q(x) d x
$$

where $q(x)$ is the risk-neutral density of the log-price $x_{T}$. Let $\zeta_{T}(u)$ be the Fourier transform of $z_{T}(k)$ :

$$
\zeta_{T}(u)=\int_{-\infty}^{\infty} e^{i u k} z_{T}(k) d k
$$

By considering a damping function $\sinh (\alpha k)$, the time value of an option follows a Fourier inversion:

$$
z_{T}(k)=\frac{1}{\sinh (\alpha k)} \frac{1}{\pi} \int_{0}^{\infty} e^{-i u k} \Upsilon_{T}(u) d u
$$

where $\Upsilon_{T}(u)=\left(\zeta_{T}(u-i \alpha)-\zeta_{T}(u+i \alpha)\right) / 2$.

The use of the FFT for calculating OTM option prices is similar to (4.8). The only differences are that they replace the multiplication by $\exp \left(-\alpha k_{u}\right)$ with a division by $\sinh (\alpha k)$ and the function call to $\psi(v)$ is replaced by a function call to $\Upsilon_{T}(u)$.

\section{Simulation Studies}

In this section, to compare across the BS, DEJD, and SVDEJD models, we analyze the probability densities of these models. Then, we analyze mainly the impact of $\rho$ and volatility of volatility $\sigma_{v}$ on option pricing under the SVDEJD model. For our FFT methods, we used $N=4096$ points in our quadrature, implying a log-strike spacing of $h=\pi / 300=0.01047$, which is adequate for practice. For the choice of the dampening coefficient in the transform of the modified call price, we used a value of $\alpha=2.55$. For the modified time value, we used $\alpha=1.55$. Other parameter values used in the computation are listed in Table 1. (We have used analytic moments to set plausible parameter values for the model. For a formal econometric estimator, one could use these moments to develop a generalized method of moments estimator within the framework of Hall and Inoue [21].) 
Table 1: Default parameters for simulation of option prices.

\begin{tabular}{lc}
\hline Parameter & Value \\
\hline Probability of upward & $p=0.6$ \\
Volatility of asset price & $\sigma=0.16$ \\
Mean of the exponential distribution of upward & $\eta_{1}=40$ \\
Mean of the exponential distribution of downward & $\eta_{2}=40$ \\
Intensity of the Poisson process & $\lambda=10$ \\
Interest rate & $r=0.05$ \\
Initial asset price & $S_{0}=100$ \\
Initial variance & $V_{0}=1$ \\
Rate of reversion & $a_{v}=0.3$ \\
Long-run variance & $\theta_{v}=0.6$ \\
Volatility of volatility & $\sigma_{v}=0.25$ \\
Correlation between returns and volatility & $\rho=-0.8$ \\
\hline
\end{tabular}

\subsection{Probability Densities under Alternative Models}

We compare the probability densities of the SVDEJD model, the BS model, and the DEJD model to verify the rationality of our model. Suppose $\varphi(u)$ is the characteristic function of $x_{T}$ and $q(x)$ the probability density of our model. From FFT algorithm, $q(x)$ can be approximated by

$$
q(x) \approx \frac{1}{\pi} \sum_{j=1}^{N} e^{-i(2 \pi / N)(j-1)(k-1)} \varphi(u) \quad k=1, \ldots, N .
$$

The density $q$ has the mean and variance given by

$$
\begin{gathered}
E_{q}(Q)=\frac{\varphi^{\prime}(0)}{i} \\
\operatorname{Var}_{q}(Q)=-\varphi^{\prime \prime}(0)+\left(\varphi^{\prime}(0)\right)^{2} .
\end{gathered}
$$

Figure 1 shows the figures of the probability density $q(x)$, compared with the normal density with the same mean and variance given by (5.2). The first figure compares the overall shapes of the densities of the SVDEJD model and the BS model, the second one details the shapes around the peak areas, and the last one shows the right tail. From Figure 1, we can see that the leptokurtic and skewness feature of the density of our model is quite evident. Moreover, additional numerical plots suggest that the feature of skewness becomes more significant if $|\rho|$ increases, which is impossible for the DEJD model.

We also compare the short-term and long-term densities of the SVDEJD model, the BS model, and the DEJD one. Figure 2 shows their densities under $T=3$ months and $T=2$ years. From Figure 2, we can see that the SVDEJD model and the DEJD model generate virtually identical densities for short-term options, with a slight departure occurring between the two densities in the upper tail. This means that differential pricing performance between the SVDEJD model and the DEJD model is unlikely to occur when they are applied to price 

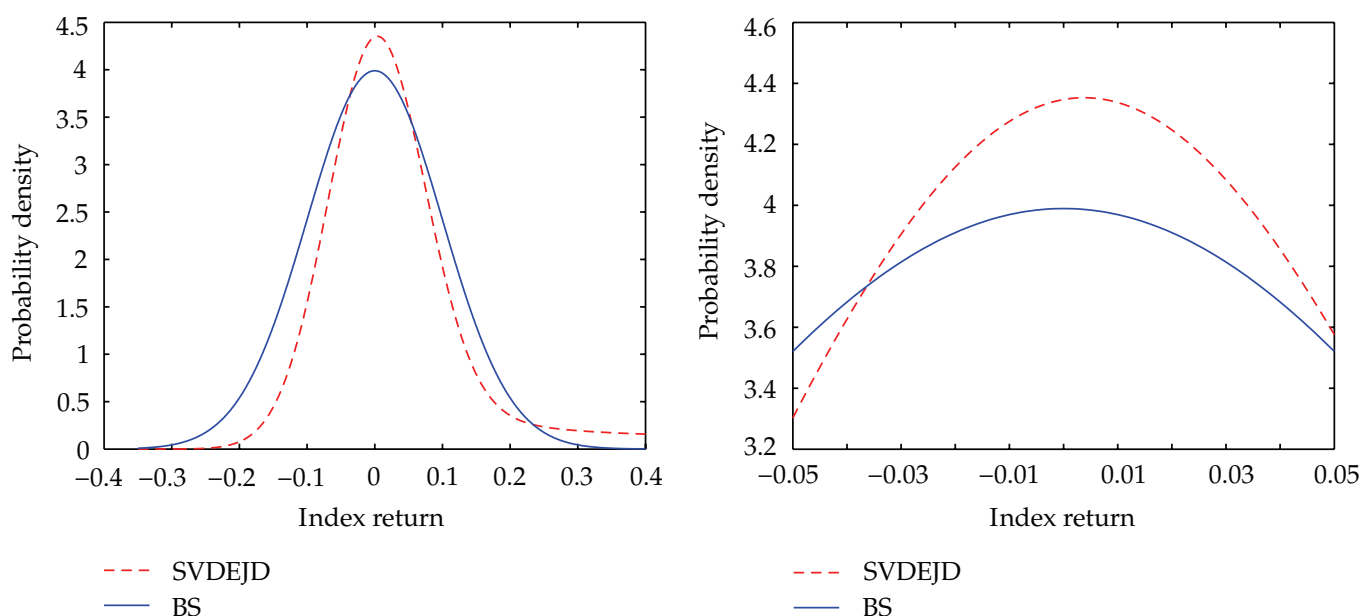

- BS

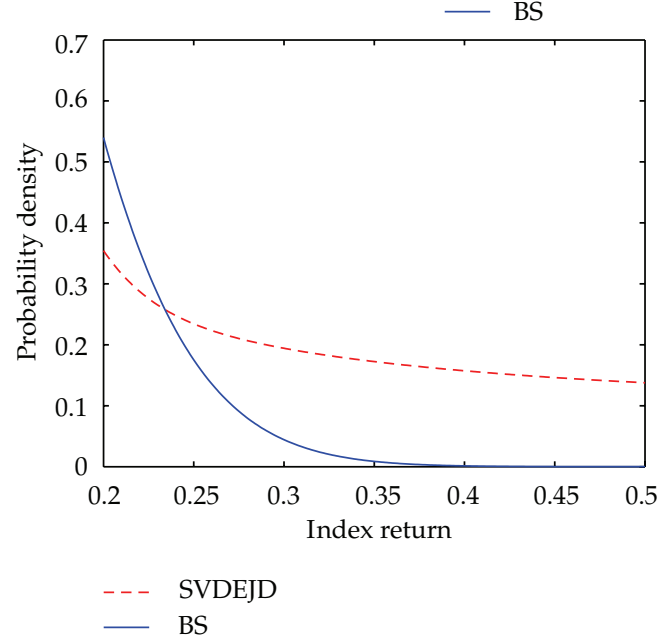

Figure 1: Comparison of the probability densities of the SVDEJD model and the BS model. Except for the maturity time $T=2$ years, the parameters used here are shown in Table 1 .

short-term OTM puts and that only when they are applied to deep ITM puts (and deep OTM calls) can differences be observed between these models. Yet, compared to the BS model density, the densities of the two models are distinctly different: they all have leptokurtic and skewness feature. Therefore, the two models can potentially correct the BS model's tendency to underprice deep OTM puts and overprice deep OTM calls. The long-term density curves in Figure 2 still show significantly different pricing structures between the BS and its two alternatives. But, more importantly, the densities of the SVDEJD model and the DEJD model also exhibit different shapes now. The SVDEJD density has higher peak and assigns more weight to both the entire lower tail and the far upper tail, but less weight to those payoffs than the DEJD.

Our simulation studies have demonstrated that the SVDEJD model has better performance than the DEJD one on pricing long-term options, while both the DEJD model and the SVDEJD model have better performance than the BS model. 

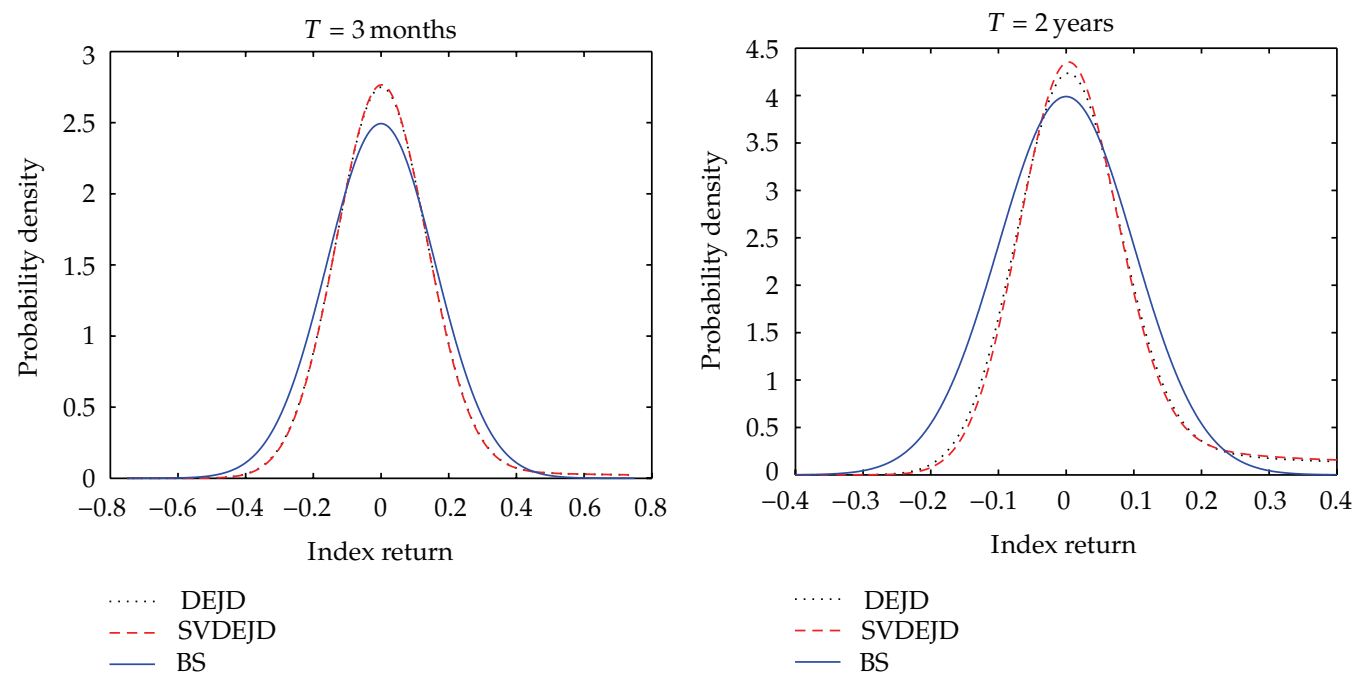

Figure 2: Comparison of the short-term and long-term probability densities of the SVDEJD model, the BS model, and the DEJD model. Except for the maturity time $T=3$ months and $T=2$ years, the parameters used here are shown in Table 1.

Table 2: The effects of volatility of volatility, exercise price $K$, maturity time $T$, and correlation $\rho$ on option values.

\begin{tabular}{lcccccc}
\hline \multirow{2}{*}{ Strike price } & \multicolumn{2}{c}{$\rho=-0.8$} & \multicolumn{2}{c}{$\rho=0$} & \multicolumn{2}{c}{$\rho=0.8$} \\
& $\sigma_{v}=0.15$ & $\sigma_{v}=0.25$ & $\sigma_{v}=0.15$ & $\sigma_{v}=0.25$ & $\sigma_{v}=0.15$ & $\sigma_{v}=0.25$ \\
\hline$T=3$ months & & & & & & \\
90 & 11.5004 & 11.5122 & 11.4827 & 11.4829 & 11.4647 & 11.4527 \\
95 & 7.5901 & 7.6000 & 7.5745 & 7.5741 & 7.5585 & 7.5472 \\
100 & 4.4325 & 4.4323 & 4.4315 & 4.4307 & 4.4303 & 4.4287 \\
105 & 2.5442 & 2.5347 & 2.5573 & 2.5567 & 2.5567 & 2.5780 \\
110 & 1.1258 & 1.1109 & 1.1476 & 1.1476 & 1.1690 & 1.1830 \\
115 & 0.5169 & 0.5043 & 0.5361 & 0.5364 & 0.5532 & 0.5681 \\
$T=2$ years & & & & & & \\
90 & 22.5229 & 22.5682 & 22.4438 & 22.4398 & 22.3592 & 22.2959 \\
95 & 19.4525 & 19.4825 & 19.3935 & 19.3872 & 19.3293 & 19.2776 \\
100 & 16.6650 & 16.6750 & 16.6337 & 16.6255 & 16.5979 & 16.5633 \\
105 & 14.3313 & 14.3225 & 14.3271 & 14.3179 & 14.3189 & 14.3018 \\
110 & 11.9850 & 11.9507 & 12.0183 & 12.0083 & 12.0476 & 12.0549 \\
115 & 10.1354 & 10.0809 & 10.1994 & 10.1896 & 10.2594 & 10.2871 \\
\hline
\end{tabular}

\subsection{Effects of the Main Parameter on Option Values}

In Table 2, we use the SVDEJD model to examine the effects of volatility of volatility $\sigma_{v}$, the correlation coefficient $\rho$, exercise price $K$, and maturity time $T$ on option values. We analyze the prices of three-month call options and two-year call options. To examine the effect of the negative correlation coefficient, we have calculated the model with $\rho=-0.8, \rho=0$, and $\rho=0.8$. The prices for three-month call options associated with volatility of volatility $\sigma_{v}=0.15$ 
and $\sigma_{v}=0.25$ are relatively close. With $\rho=-0.8$, the largest price difference is 0.0149 ; with $\rho=0$, the largest price difference is only 0.0008 ; with $\rho=0.8$, the largest price difference is 0.0213 . However, the difference is significantly larger when longer-time horizons such as two-year call options are valued. With $\rho=-0.8$, the largest price difference is an increase on 0.0149 to 0.0545 and the effect of volatility of volatility is an increase for ITM calls and a decrease for OTM calls. With $\rho=0$, the largest price difference is increase of 0.0008 to 0.01 and the effect of volatility of volatility is a small decreas that is negligible for option values. With $\rho=0.8$, the largest price difference is an increase of 0.0213 to 0.0633 and the effect of volatility of volatility is a decrease for ITM and ATM calls and an increase for OTM calls. The correlation parameter $\rho$ has several effects depending on the relation between the strike price and the current stock price. A negative $\rho$ tends to produce higher values for ITM calls and lower values for OTM money calls.

We have also compared the model with the BS model, which can be interpreted as a first-order approximation with no jumps, and $\rho=0$. A common practice is to set the implied volatility in the BS model so that the model matches the price for the option with a strike price closest to the current stock price. For some comparisons not reported here, we have set the implied volatility in the BS model so that it matches the price generated by the stochastic volatility model for an ATM option. The BS implied volatility is very close to the expected volatility under the risk-neutral distribution when short-term options are valued. When longer-term options are used, there is a significant difference between the BS implied volatility and the expected volatility. As an approximation, the BS model tends to undervalue ITM calls and overvalue OTM calls.

\section{Conclusion}

The SVDEJD model incorporates several important features of stock returns. We derive a closed-form solution for European call options in the model by using the martingale method, Fourier inversion transform formula, and Feynman-Kac theorem. Using FFT, we obtain fast and accurate numerical solution to European option under the model. The comparison of densities of the alternative models shows that the SVDEJD model has better pricing performance on long-time options. An analysis of the model reveals that volatility of volatility $\sigma_{v}$ and the correlation coefficient $\rho$ have significant impact on option values, especially long-time option, stock returns are negatively correlated with volatility, and these negative correlations are important for option valuation.

\section{Appendix}

\section{A.1. Matlab Codes for ITM and ATM Options Pricing by FFT}

$$
\begin{aligned}
& \text { function } C V=\operatorname{inSVDexpJ}(\text { ata1, ata2, lamta, sigma, thetav, alphav, } \\
& \text { rho, sigmav, r, p, s0, v0, strike, T) } \\
& \mathrm{x} 0=\log (\mathrm{s} 0) \\
& \text { alpha }=2.55 \\
& \mathrm{~N}=4096 \\
& \mathrm{c}=600
\end{aligned}
$$




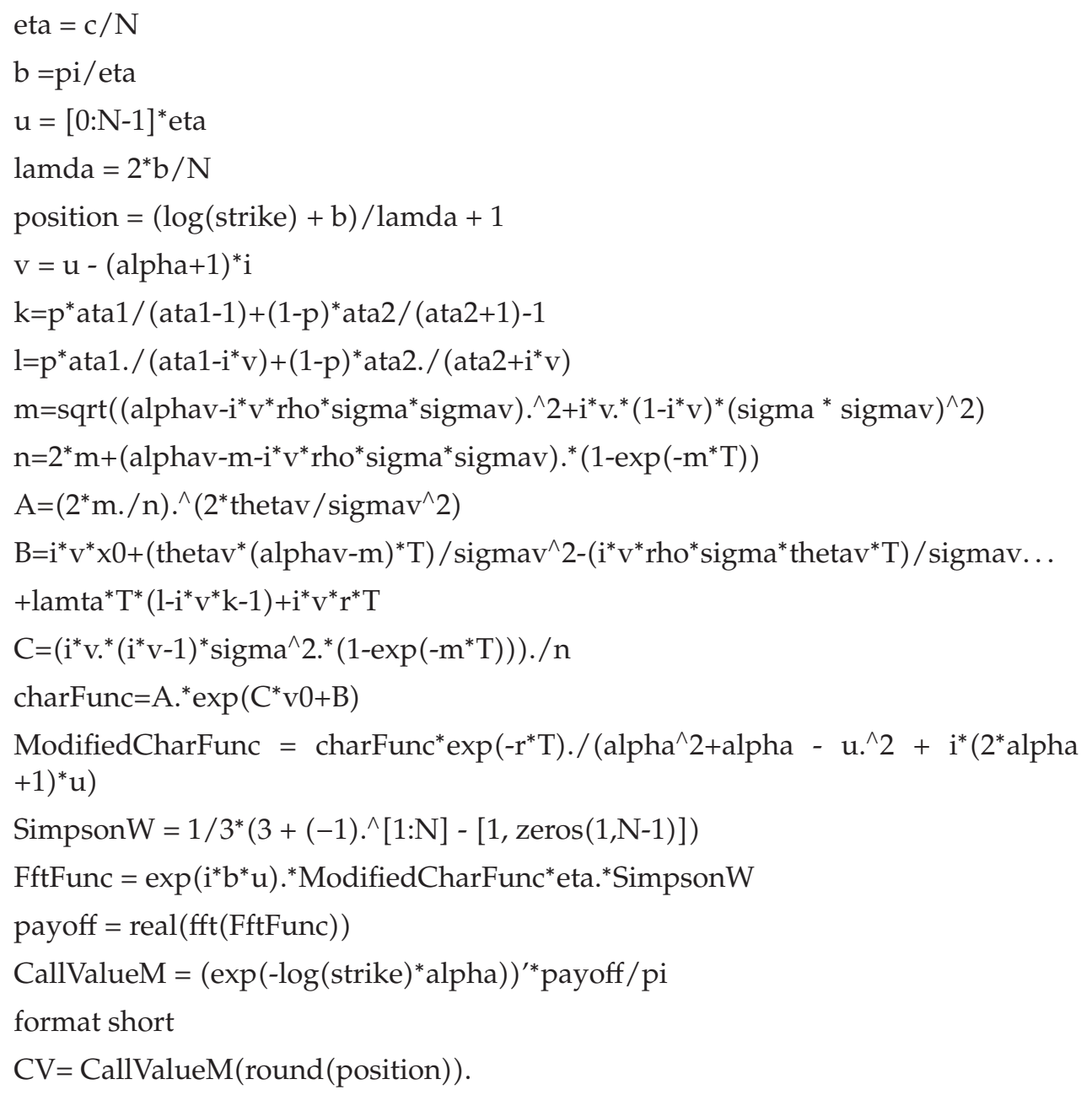

\section{A.2. Matlab Codes for OTM Options Pricing by FFT}

function CV=outSVDexpJ(ata1,ata2,lamta,sigma, thetav,alphav, rho,sigmav,r,p,s0,v0,strike, $\mathrm{T}$ )

$\mathrm{x} 0=\log (\mathrm{s} 0)$

alpha $=1.55$

$\mathrm{N}=4096$

$c=600$

eta $=\mathrm{c} / \mathrm{N}$

$\mathrm{b}=\mathrm{pi} / \mathrm{eta}$

$\mathrm{u}=[0: \mathrm{N}-1]^{*}$ eta

lamda $=2 * \mathrm{~b} / \mathrm{N}$

position $=(\log ($ strike $)+b) / \operatorname{lamda}+1$

$\mathrm{w} 1=\mathrm{u}-\mathrm{i}^{*}$ alpha 


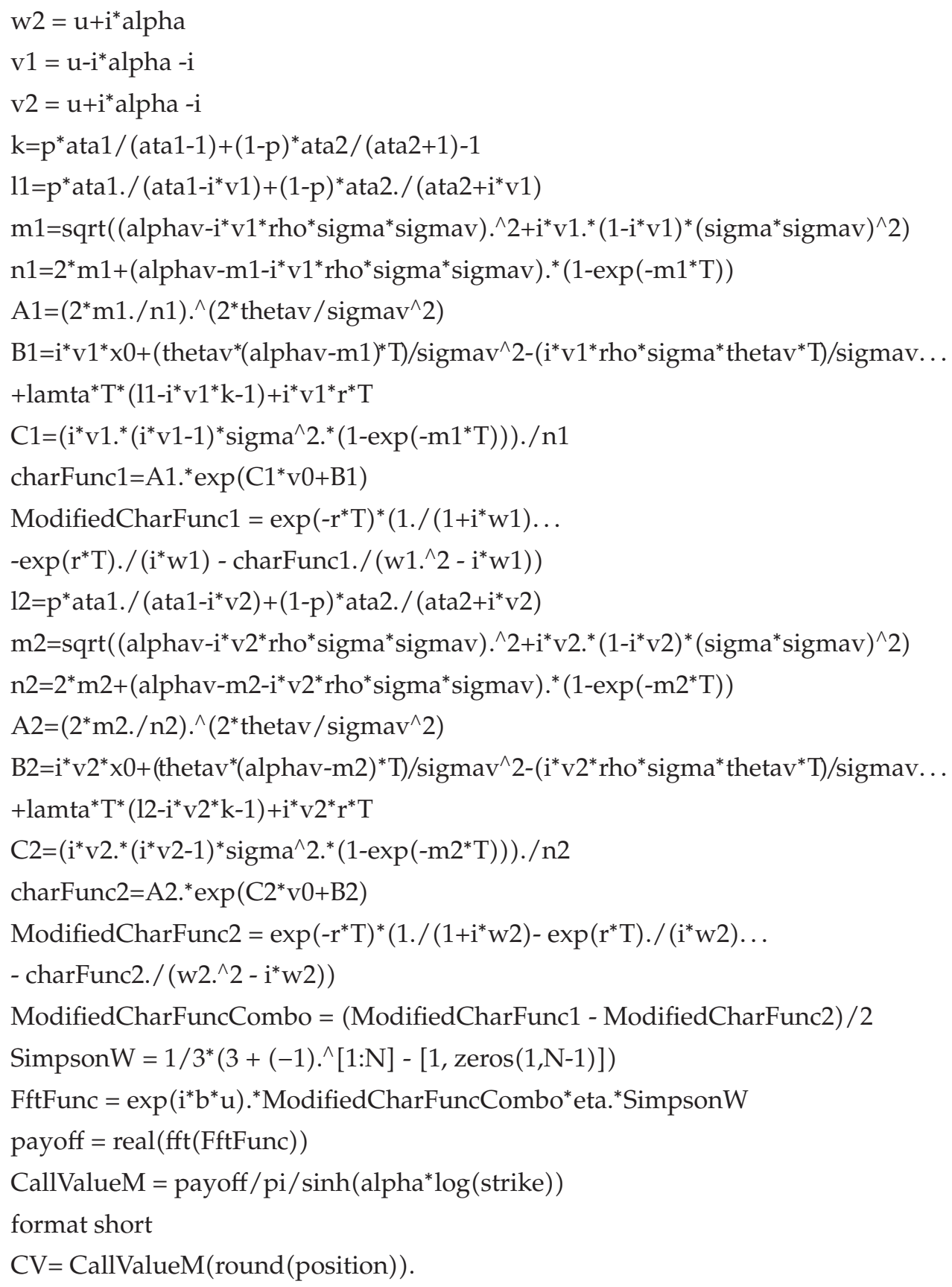

\section{Acknowledgments}

This work is supported by the National Natural Science Foundation of China (Grant no. 11171266) and the Science Plan Foundation of the Education Bureau of Shanxi Province (nos. 11JK0491 and 11JK0493). The authors are grateful to the reviewers as well as the editor for their valuable comments and suggestions, which led to a substantial improvement of the paper. 


\section{References}

[1] F. Black and M. S. Scholes, "The pricing of options and corporate liabilities," Journal of Political Economy, vol. 81, no. 3, pp. 637-654, 1973.

[2] R. C. Merton, "Option pricing when underlying stock returns are discontinuous," Journal of Financial Economics, vol. 3, no. 1-2, pp. 125-144, 1976.

[3] R. Cont, "Empirical properties of asset returns: stylized facts and statistical issues," Quantitative Finance, vol. 1, no. 2, pp. 223-236, 2001.

[4] R. Cont, J. da Fonseca, and V. Durrleman, "Stochastic models of implied volatility surfaces," Economic Notes, vol. 31, no. 2, pp. 361-377, 2002.

[5] E. Eberlein and U. Keller, "Hyperbolic distributions in finance," Bernoulli, vol. 1, no. 3, pp. 281-299, 1995.

[6] D. B. Madan, P. P. Carr, and E. C. Chang, "The variance gamma process and option pricing," European Finance Review, vol. 2, no. 1, pp. 79-105, 1998.

[7] D. Duffie, J. Pan, and K. Singleton, "Transform analysis and asset pricing for affine jump-diffusions," Econometrica, vol. 68, no. 6, pp. 1343-1376, 2000.

[8] L. H. Steven, "A closed-form solution for options with stochastic volatility with applications to bond and currency options," Review of Financial Studies, vol. 6, no. 2, pp. 327-343, 1993.

[9] D. S. Bates, "Jumps and stochastic volatility: exchange rate processes implicit in deutsche mark options," Review of Financial Studies, vol. 9, no. 1, pp. 69-107, 1996.

[10] J. Keppo, X. Meng, S. Shive, and M. Sullivan, "Modelling and hedging options under stochastic pricing parameters," in Proceedings of the Industrial and Operations Engineering, University of Michigan at Ann Arbor, 2003.

[11] S. G. Kou, “A jump-diffusion model for option pricing,” Management Science, vol. 48, no. 8, pp. 10861101, 2002.

[12] S. G. Kou and H. Wang, "Option pricing under a double exponential jump diffusion model," Management Science, vol. 50, no. 9, pp. 1178-1192, 2004.

[13] J. R. Birge and V. Linetsky, Handbooks in Operations Research and Management Science, vol. 15, NorthHolland, 2008.

[14] G. Bakshi, C. Cao, and Z. Chen, "Pricing and hedging long-term options," Journal of Econometrics, vol. 94, no. 1-2, pp. 277-318, 2000.

[15] T. W. Epps, Pricing Derivative Securities, World Scientific, Singapore, 2nd edition, 2007.

[16] P. P. Carr and D. B. Madan, "Option valuation using the fast Fourier transform," Journal of Computational Finance, vol. 2, no. 4, pp. 61-73, 1999.

[17] P. P. Carr and L. Wu, "Time-changed Lévy processes and option pricing," Journal of Financial Economics, vol. 71, no. 1, pp. 113-141, 2004.

[18] T. R. Hurd and Z. Zhou, "A Fourier transform method for spread option pricing," SIAM Journal on Financial Mathematics, vol. 1, pp. 142-157, 2010.

[19] M. Schmelzle, “Option pricing formulae using Fourier transform: theory and application," 2010, http://pfadintegral.com .

[20] V. Naik and M. Lee, "General equilibrium pricing of options on the market portfolio with discontinuous returns," Review of Financial Studies, vol. 3, no. 4, pp. 493-521, 1990.

[21] A. R. Hall and A. Inoue, "The large sample behaviour of the generalized method of moments estimator in misspecified models," Journal of Econometrics, vol. 114, no. 2, pp. 361-394, 2003. 


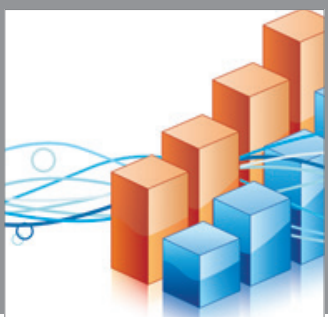

Advances in

Operations Research

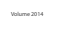

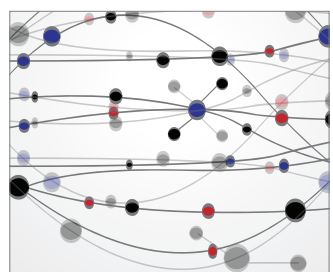

\section{The Scientific} World Journal
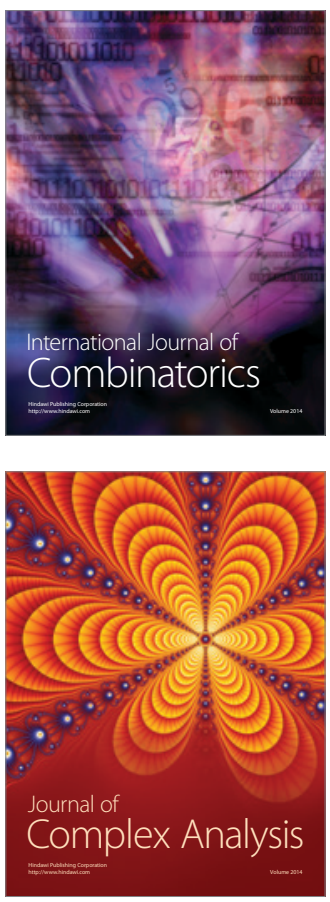

International Journal of

Mathematics and

Mathematical

Sciences
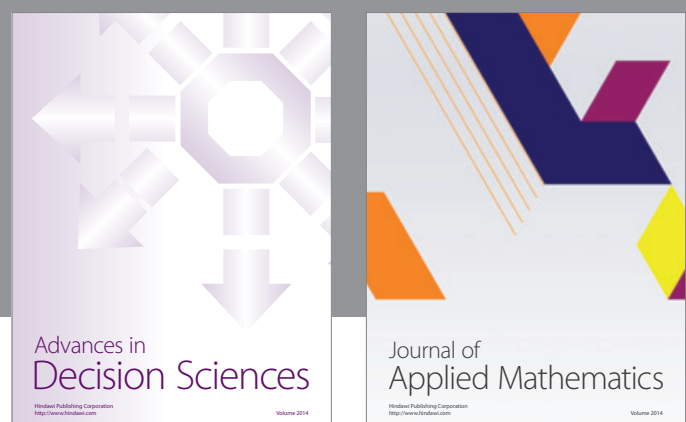

Journal of

Applied Mathematics
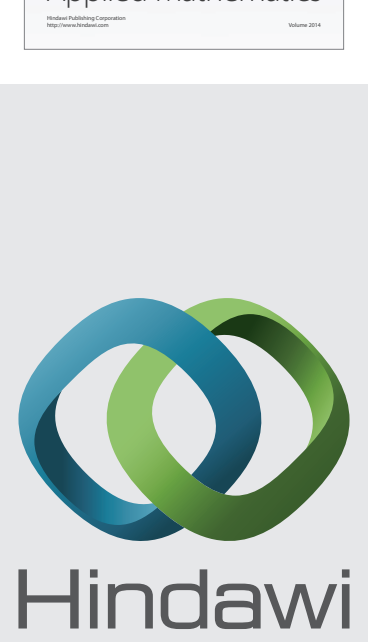

Submit your manuscripts at http://www.hindawi.com
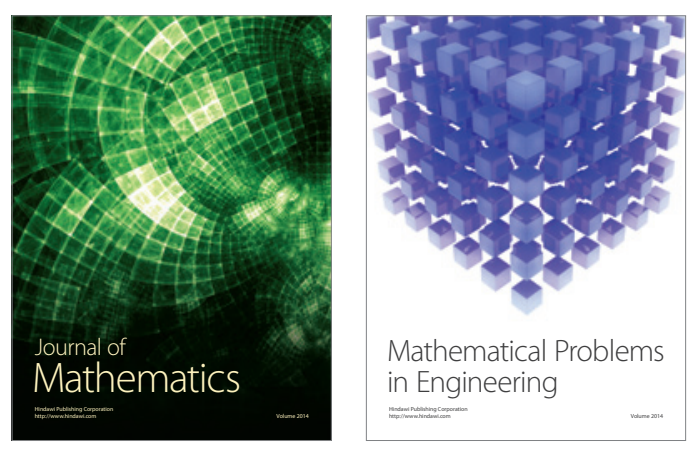

Mathematical Problems in Engineering
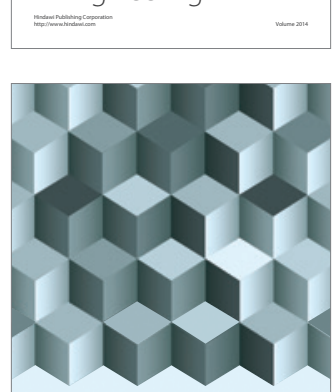

Journal of

Function Spaces
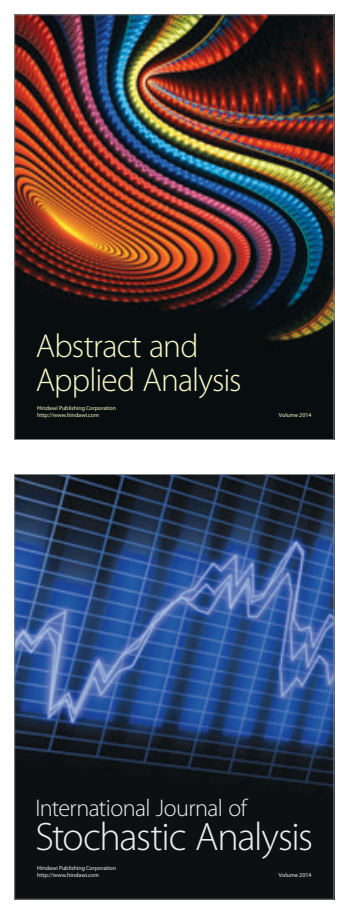

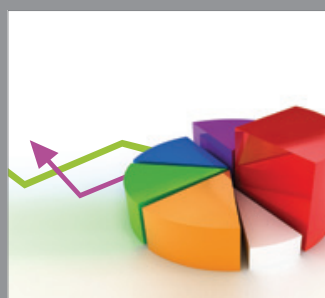

ournal of

Probability and Statistics

Promensencen
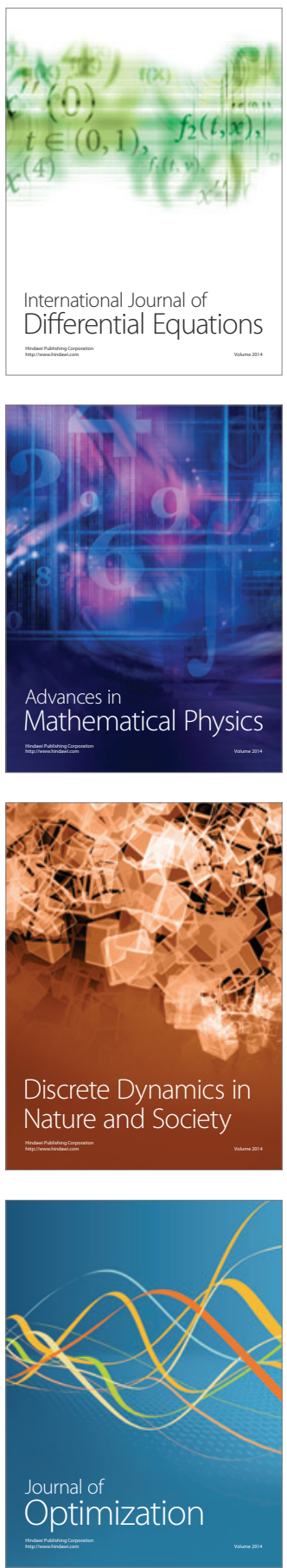